\title{
Determination of Critical Micelle Concentration of Sodium Dodecyl Sulfate in Magnrtic Water Medium by Electrical Conductivity
}

\author{
Marwa Mohammed Ali Al - Sayegh Dr. Amer Abdel Hamed AL Hatem \\ Department of Chemistry / College of Education for Girls \\ University of Mosul
}

\section{Marwamohammed997788@gmail.com}

DOI: $10.33899 /$ edusj.2019.162967

\section{Received}

27 / 01 / 2019 amer.alhatem@gmail.com

\author{
C.27/01/2019
}

Accepted

$10 / 04 / 2019$

\begin{abstract}
Anionic surfactant was investigated in magnetic water medium using conductometric technique. The critical micelle concentration (CMC) was determined both in absence and presence of different concentration percentages of short alcohol chains in a temperature range $(293.15-308.15 \mathrm{~K})$. The thermodynamic parameters

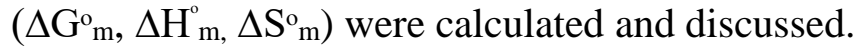

Key words: Surfactants, Conductivity, Magnatic Water. 
تقدير تركيز ألمذيل الحرج (CMC) لمركب سلفات الدوديسيل في الوسط المائي الممغط باستخدام تقنية التوصيل الكهربائي

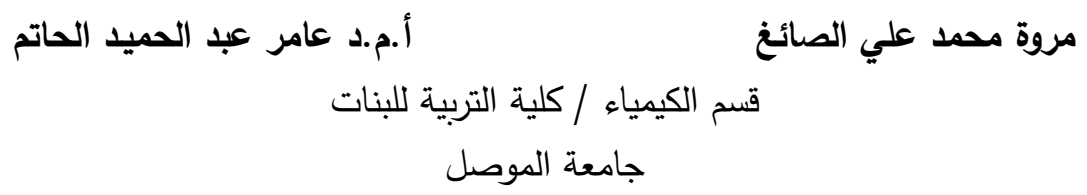

Marwamohammed997788@gmail.com

DOI: 10.33899/edusj.2019.162967

القبول

2019 / 04 / 10
الاستلام

2019 / 01 / 27

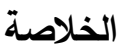

تمت دراسة المواد الفعالة سطحياً الايون السالب (SDS) في الوسط المائي الممغنط باستخدام تقنية التوصيل الكهربائي، كما تم تقدير تراكيز المذيل الحرج CMC لهذا المكون لوحده أولاً وبوجود عدة تراكيز مئوية من الكحولات قصيرة السلسلة، وتمت دراسة تأثير درجات الحرارة في المدى 293.15-308.15)K على تلى تقدير

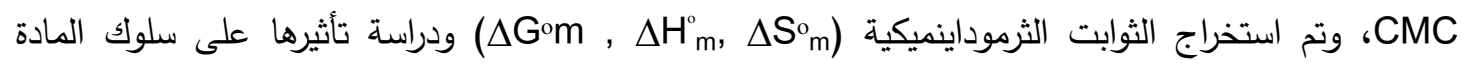
الفعالة الأيونية السالبة على تقدير CMC CMC للمركب قيد الدرس.

الكلمات المفتاحية: المواد الفعالة سطحيا، التوصيلية الكهربائية، الماء الممغنط.

Introduction

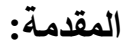

يعتبر مركب SDS من المركبات الفعالة سطحياً وهو من النوع الايون السالب الحاوي على مجموعة

قطبية أيونية (المحبة للماء) مكونة من مجموعة كبريتات سالبة الثحنة (-OSO dodecyl sulfate

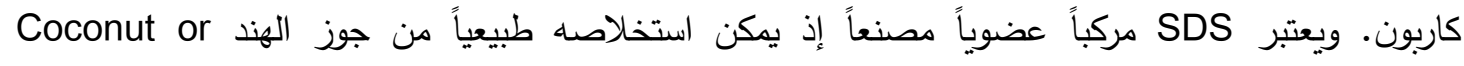
(Karnel) Oil عن مسحوق أبيض اللون خالي من أية شوائب له الصيغة الكيميائية الأتية

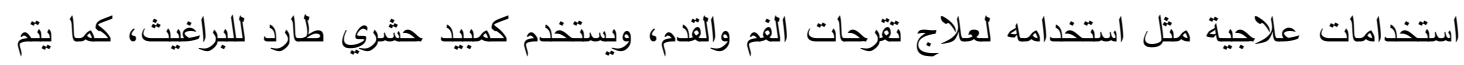

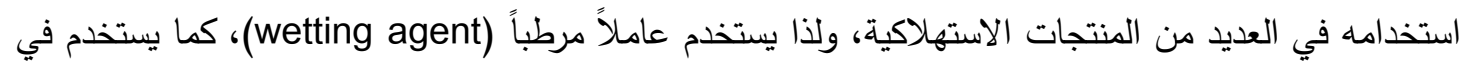

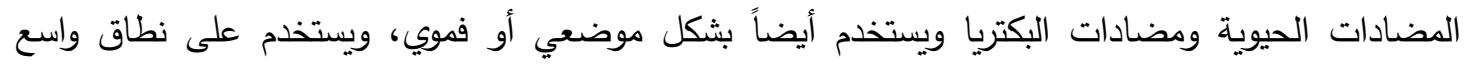

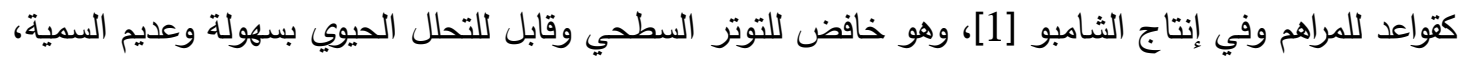
وله استخدامات أخرى كثيرة جداً لامجال لذكرها، لذلك واعتماداً على أهميته فقد تتاول الباحثون هذا التها المركب

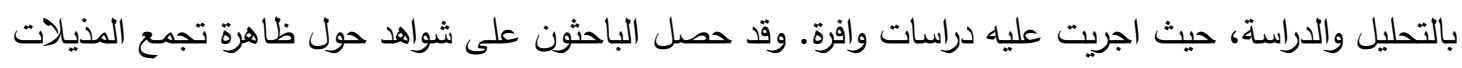

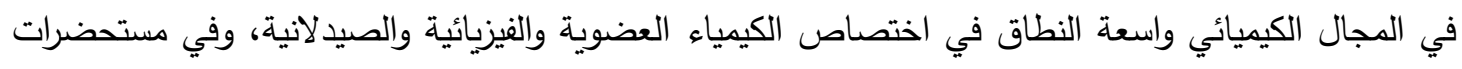

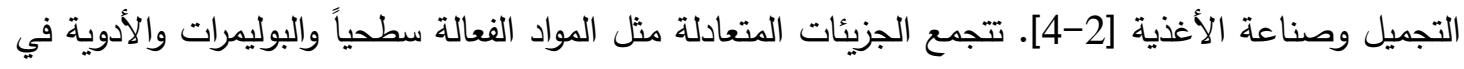
المحاليلِ المائية على السطح البيني، وهو الحد الفاصل بين طورين لإين بطريقة ذاتية ومنتظمة، لتكون ما يطلق عليها المذيلات (Micelles). يبقى تركيز الوحدات البنائية للمواد الفعالة (monomers of surfactants) ثابتاً بعد بلئل 
تخطي قيمة التركيز المذيل الحرج (CMC)، ولا يتغير التركيز بإضافة المادة الفعالة، ولكن الذي يتغير هو

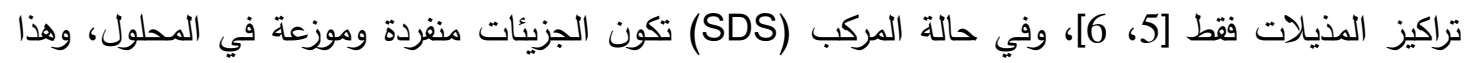

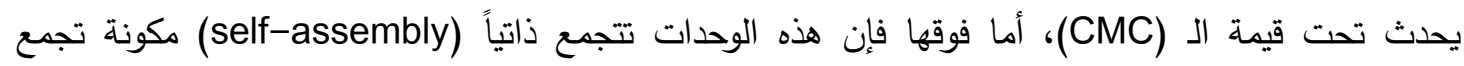

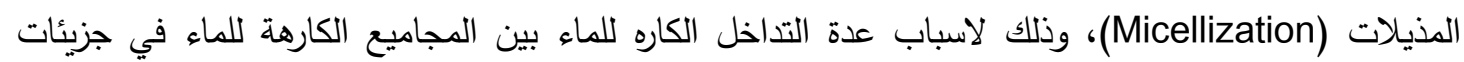
SDS

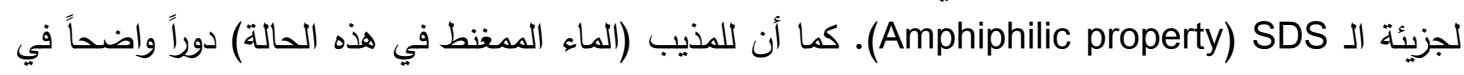

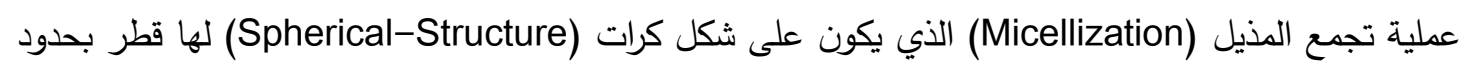
(5nm)

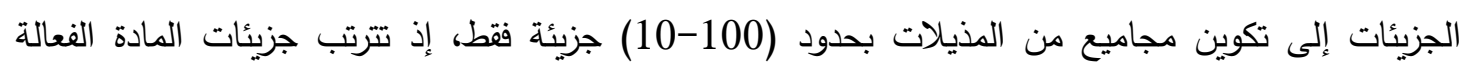

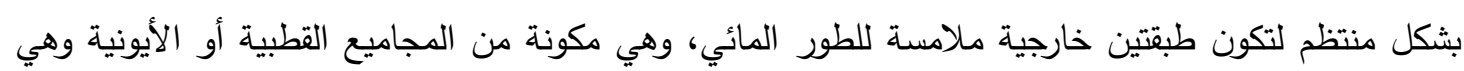
مجموعة (OSO

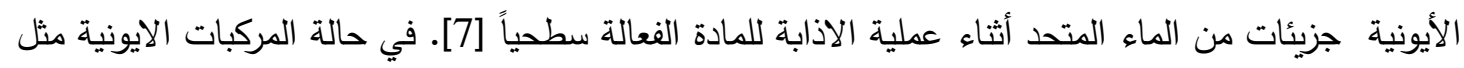
ذات الصفة المتعادلة (amphiphillic molecules) تمثل نوعية منفردة من المواد الفعالة سطحياً، ومجالاً لاراسة الجزيئات المتعادلة الحاوية على كلا النوعين من المجاميع القطبية وغير القطبية. ويبرز التأثير

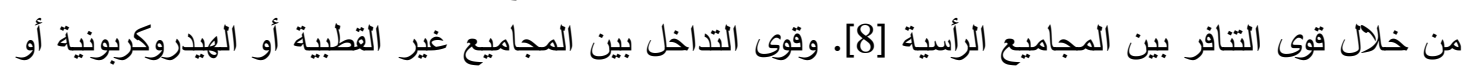

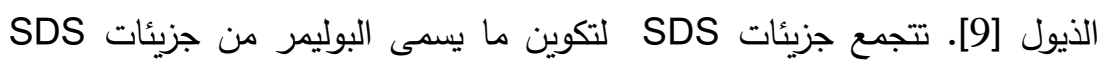
$n S \leftrightarrow S n \quad(1)$

إذ إن n تمثل عدد الوحدات البنائية للبوليمر الموجود في المحلول، أما Sn. فتمثل المادة الفعالة سطحياً

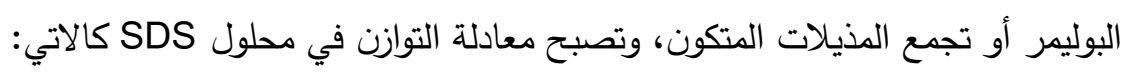

$\mathrm{K}_{\mathrm{m}}=\frac{\left[s_{n}\right]}{[S]_{n}}$

إذ إن Km يمثل ثابت التوازن المذيلات، و[S] يمثل تركيز المادة الفعالة سطحيا و[S] تركيز تجمع المذيل، و(n) يمثل عدد الوحدات البنائية للتجمع أو ما يسمى بعدد التجميع من المعادلة (2) يمكن حساب الطاقة الحرة $\Delta \mathrm{G}^{\circ} \mathrm{m}=-\mathrm{RT} \operatorname{Ln} \mathrm{K}_{\mathrm{m}}$ لهذا التجمع باستخدام المعادلة أدناه:

$$
=-R T L n\left[\mathrm{~S}_{\mathrm{n}}\right]+\mathrm{nRTLn}[\mathrm{S}]_{\mathrm{n}}
$$

إذ إن R تمثل ثابت الغاز ، وT درجة الحرارة المطلقة، ويمكن حساب الفرق في الطاقة الحرة للمول الواحد من $\mathrm{G}^{0}{ }_{\mathrm{m}}=-\frac{\mathrm{RT}}{\mathrm{n}} \operatorname{Ln} \Delta\left[\mathrm{S}_{\mathrm{n}}\right]+\mathrm{RTLn}[\mathrm{S}]$ المذيلات كالاتي: كما يمكن حساب المحتوى الحراري والانتروبي باستخدام المعادلات الاتية:

$\Delta \mathrm{H}_{\text {mic. }}^{0}=-\mathrm{RT}^{2} \mathrm{dln} \mathrm{CMC} / \mathrm{dT}$

$\Delta \mathrm{G}_{\text {mic. }}^{0}=\Delta \mathrm{H}_{\text {mic. }}^{0} \mathrm{~T} \Delta \mathrm{S}_{\text {mic. }}^{0}$

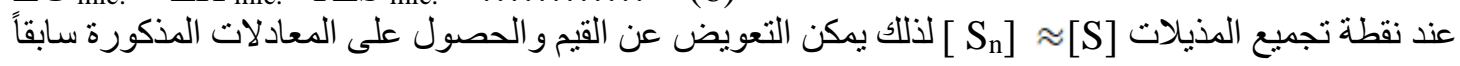

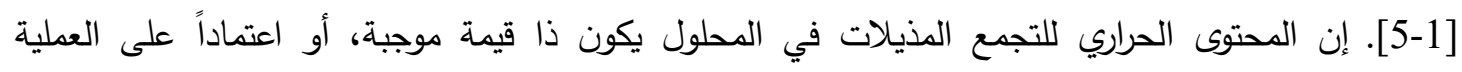

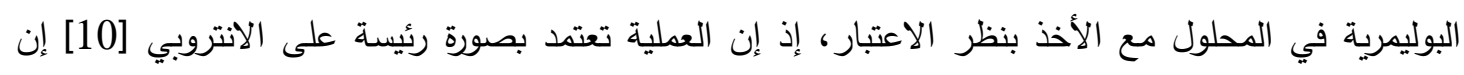

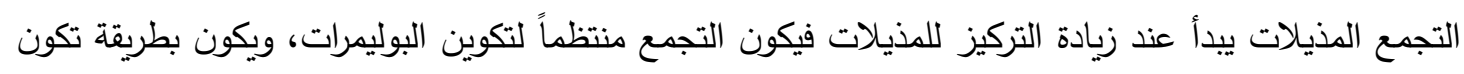
فيها المجاميع القطبية نحو الماء، أما غير قطبية (العضوية) فتكون نحو الداخل، وعند التراكيز الواطئة تتواجد 
جزيئات المذيلات أو المواد الفعالة سطحياً بشكل مستقل في المحلول ومرتبة بشكل منتظم على السطح البيني بشكل منفصل، ويكون التوتر السطحي على حاله بقيمة معينة عند زيادة تركيز المادة

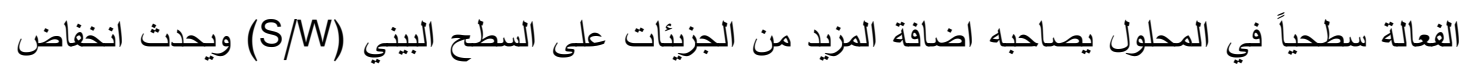
كبير في التوتر السطحي الى حالة الوصول لحالة الاشباع في السطح البيني عندها يبدا تكون الدذيلات

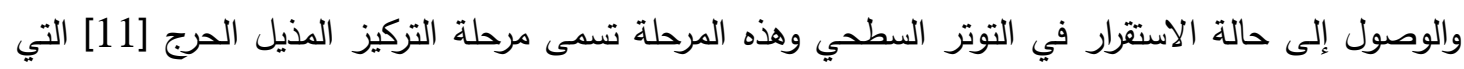

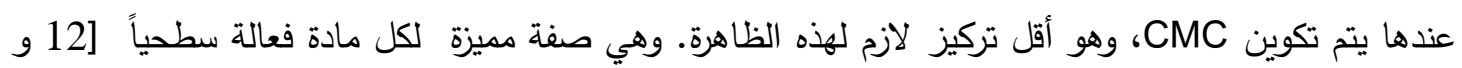

Materials and Methods

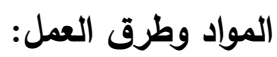

إن جميع المواد الكيميائية المستخدمة مجهزة من شركة (Oxford و و Didactic و و Scharlau)، بالإضافة الى استخدام الكحولات، وقد تم استخدام الماء المعالج مغناطيسيا مذيباً عند اجراء قياسات التوصيل الكهربائي.

\section{Conductivity of Water}

توصيلية الماء

إن نقاوة الماء مهمة جداً لكون الماء مذيباً نموذجياً لأغلب الأكتروليتات التي يتم تقديرها فضلاً عن في فاء

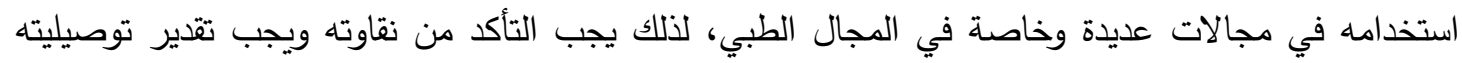

\section{Purity of Water}

نقاوة الماء

إن أفضل طريقة للحصول على ماء عالي النقاوة إضافة كمية قليلة من برمنكنات البوتاسيوم وذلك

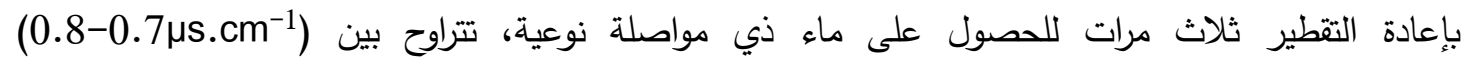

طريقة عمل الماء الممغنط:

يوضح الثكل (1) طريقة تحضير الماء الممغنط يتم تحضير الماء المغنط وذلك بملئ زجاجة بالماء

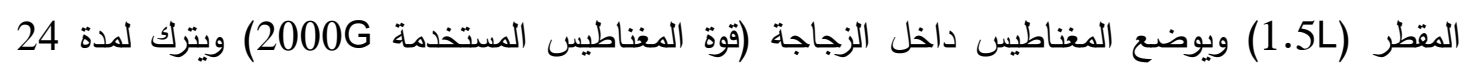

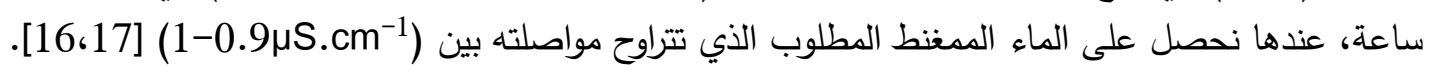

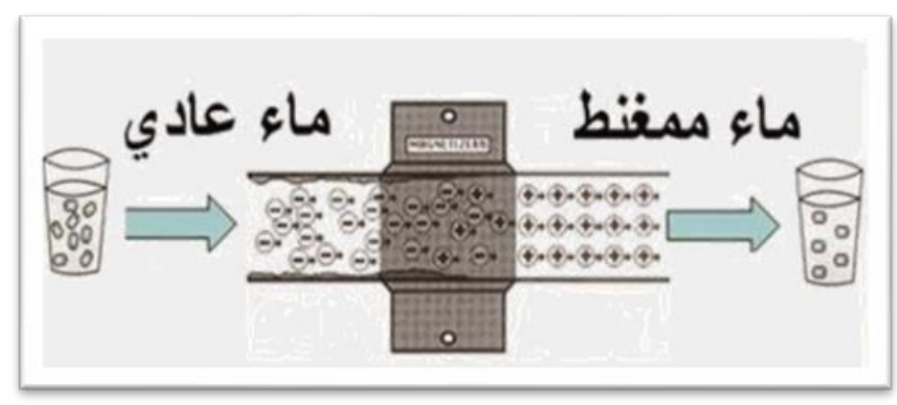

شكل (1): طريقة الحصول على الماء الممغنط

Conductivity measurement solutions

محاليل قياسات التوصيلية 


\section{Preparation of anionic SDS solution}

تحضير محلول الايون السالب SDS

حضر (250 ml) بتركيز (0.1M) كمحلول أصل Stock solution) من ال SDS Sوذلك بإذابة

(7.209 g) من المادة في أقل كمية من الماء الممغنط، ثم إكمال الحجم إلى (250 ml) باستخدام قنينة

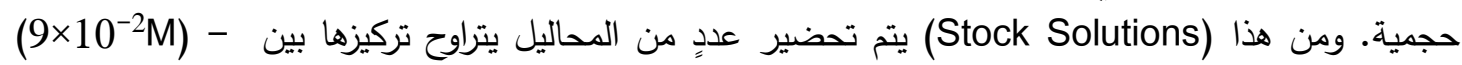
باستخدام الماء الممغنط مذيباً.

تأثثير وجود الكحولات للمادة الفعالة سطحيا السالبة SDS:

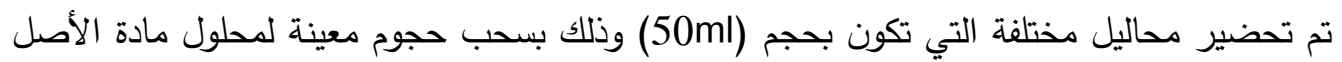

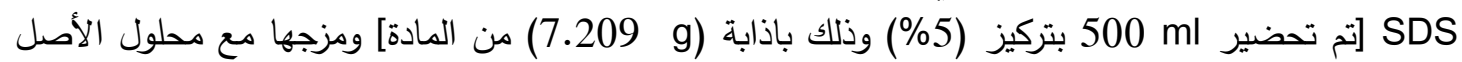

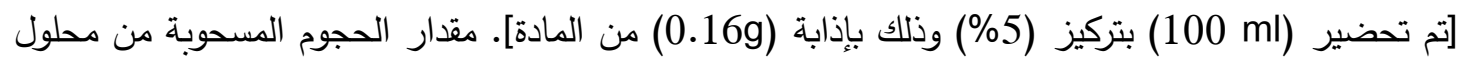
الأصل لكلتا المادتين تم توضيحهما في الجدول (1).

\begin{tabular}{|c|c|c|c|c|c|c|c|c|c|c|}
\hline SDS (ml) & 49.5 & 49.0 & 48.5 & 48.0 & 47.5 & 47.0 & 46.5 & 46.0 & 45.5 & 45.0 \\
\hline |الكحول(ml) & 0.5 & 1.0 & 1.5 & 2.0 & 2.5 & 3.0 & 3.5 & 4.0 & 4.5 & 5.0 \\
\hline
\end{tabular}

دراسة توصيلية المادة الفعالة سطحياً SDS (الايون السالب) وتحديد قيمة CMC للمركب:

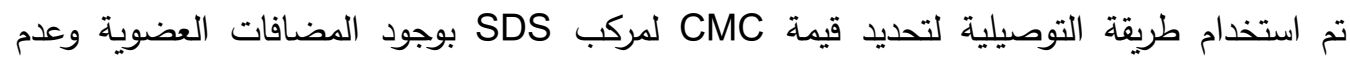

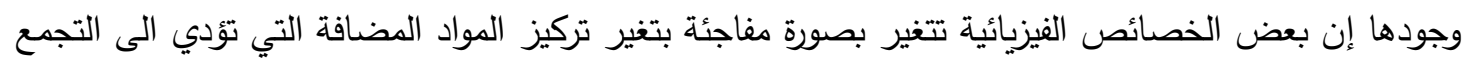

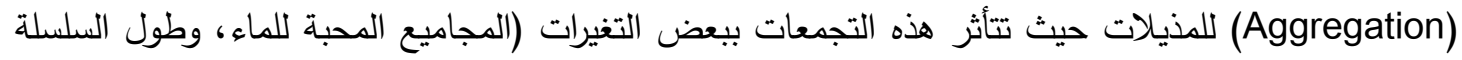

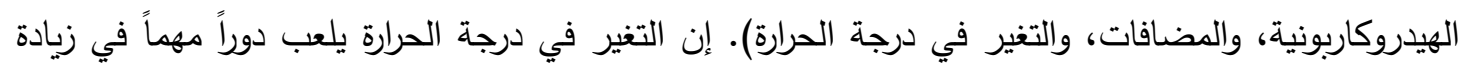

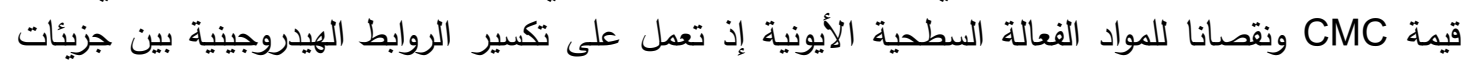

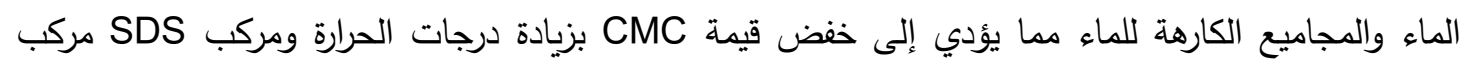

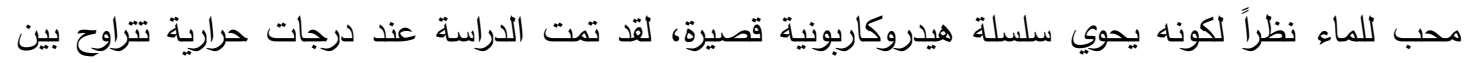

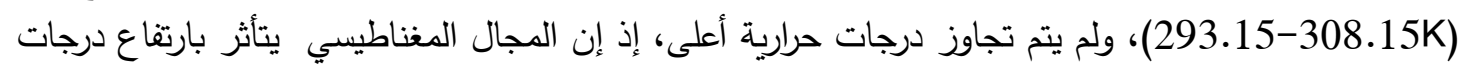

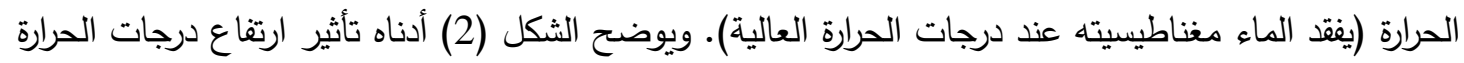
على قيمة CMC لمركب SDS في المحلول المائي الممغنط من خلال قياسات التوصيلية الكهربائية.

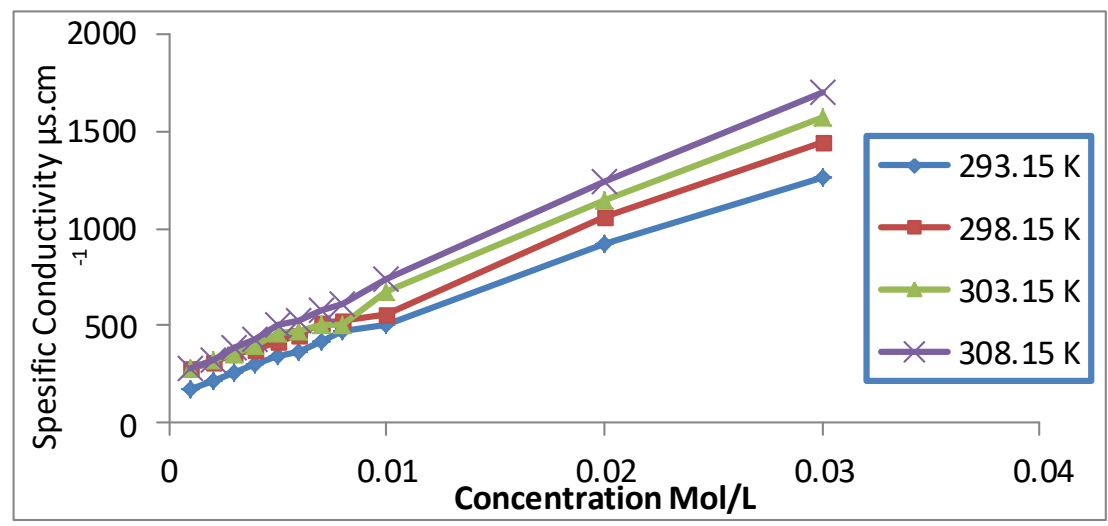

الثكل (2): قياس التوصيلية النوعية مقابل التركيز للححلول المائي المفتط للمادة الفعالة سطيا SDS عذ درجات حرارية مختلفة

إذ الذي تم حسابه وتحديده من خلال الرسم البياني يطابق إلى حد كبير ما تم إيجاده في الأدبيات المنشورة (18). 


\begin{tabular}{||c|c|c||}
\hline \multirow{2}{*}{ T(K) } & \multicolumn{2}{|c|}{ SDS } \\
& CMC (M) & Ln CMC \\
\hline 293.15 & 0.008 & -4.8283 \\
\hline 298.15 & 0.0072 & -4.9336 \\
\hline 303.15 & 0.0051 & -5.2785 \\
\hline 308.15 & 0.0032 & -5.7446 \\
\hline
\end{tabular}

SDS عند درجات حرارية مختلفة للمادة الفعالة سطحياً CMC (2): قيم

وبرسم العلاقة ما بين درجة الحرارة Ln CMC ، T(K) إذ يعطي الميل خطاً مستقيماً الذي نحسب منه قيمة الانثالبي

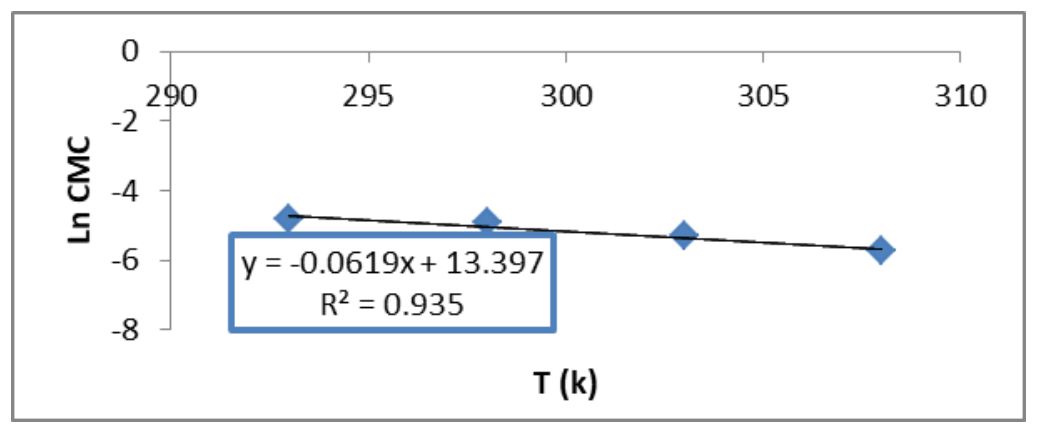

SDS قالثكل (3): قيم LnCMC مقابل درجات الحرارة المختلفة للمحلول المائي الممغط للمادة الفعالة سطحياً

وقد تم حساب المتغيرات الثرموداينمكية في مدى من الدرجات الحرارية تتراوح (308.15K- 293.15) باستخدام المعادلات (6,5,4,3) ويبين الجدول (3) قيم المتغيرات الثرموداينمكية المستحصل عليها من دراسة المحاليل المائية للمادة الفعالة سطحياً

\begin{tabular}{|c|c|c|c|c|}
\hline \multicolumn{5}{|c|}{ المادة الفعالة سطيا SDS } \\
\hline المتغيرات الثرموداينمكية & $T=293.15 K$ & $T=98.15 K$ & $\mathrm{~T}=303.15 \mathrm{~K}$ & $\mathrm{~T}=308.15 \mathrm{~K}$ \\
\hline$\Delta G_{\mathrm{m}}^{\circ}\left(\mathrm{KJ} \cdot \mathrm{Mol}^{-1}\right)$ & -11.7617 & -12.2233 & -13.2972 & -14.7102 \\
\hline$\Delta \mathbf{H}_{\mathrm{m}}^{\circ}\left(\mathrm{KJ} \cdot \mathrm{Mol}^{-1}\right)$ & 44.1810 & 45.7017 & 47.2482 & 48.8204 \\
\hline$\Delta \mathbf{S}_{\mathrm{m}}^{\mathrm{o}}\left(\mathrm{KJ} \mathrm{Mol}^{-1} \cdot \mathrm{K}^{-1}\right)$ & 0.1909 & 0.1943 & 0.1998 & 0.2062 \\
\hline
\end{tabular}
الجدول (3): قيم المتغيرات الثرموداينمكية للمادة الفعالة سطحياً SDS عند درجات حرارية مختلفة

إذ رسمت المتغيرات الثرموداينمكية للدحاليل المائية للمادة الفعالة سطحياً SDS عند درجات حرارية مختلفة كما هو موضتح في الثكل (4): 

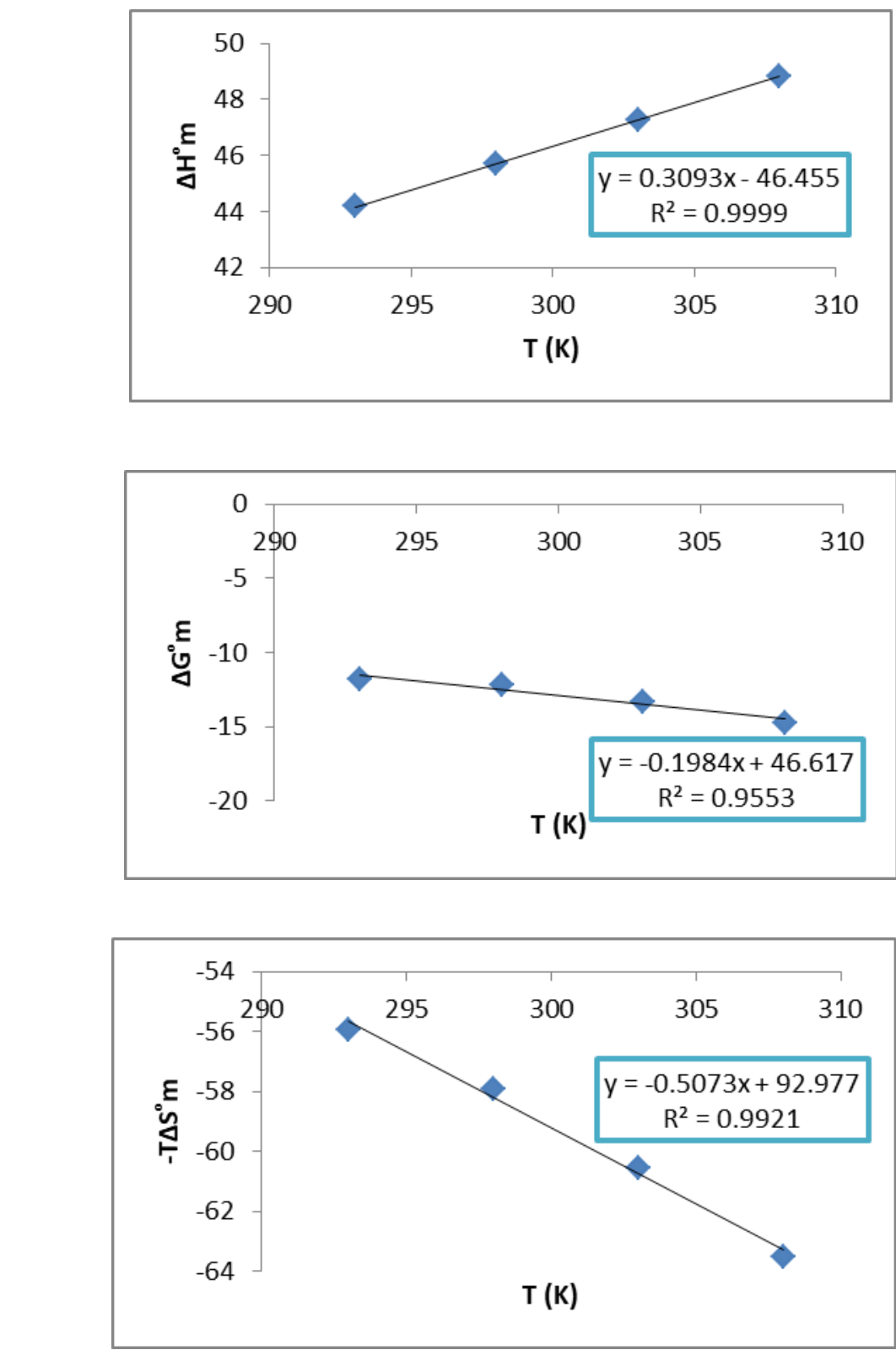

الثكل (4): العلاقة بين قيم المتغيرات الثرموداينمكية لمحاليل المائية المفتطة عند درجات حرارية مختلفة SDS Sلمادة الفعالة سطحياً

تأثير إضافة الكحولات (الميثانول، الايثانول، والبروبانول):

تستخدم الكحولات وخاصة ذات السلاسل القصيرة مثل (Methanol,Ethanol, and n-Propanol) التي لها القدرة والقابلية على تغيير صفات بعض المحاليل للمواد الفعالة سطحياً عند امتزاجها بنسب مختلفة، ومن اهم هذه الاسباب المؤدية إلى تغيير الصفات هي الروابط الهيدروجينية، والتداخلات الكارهة للماء، والارتباط بزيئات الماء (hydration) [19] أي تحدث عملية التميؤ وظهور مركبات جديدة لها صلة وثيقة بالمركبات الكحولية قيد الدراسة، كما يؤدي ذلك الى التغيير في الكثير من الصفات الفيزيائية والكيميائية مثل اللزوجة، والكثافة، والمواصلة الكهربائية، والثد السطحي، ...الخ، إن تأثير إضافة الكحولات المختلفة عند درجات حرارية

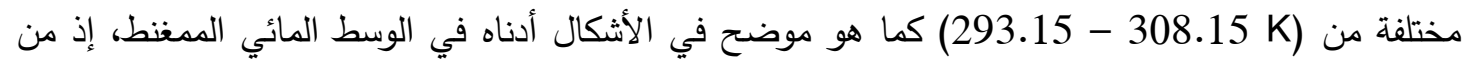
المعروف ان الكحولات المضافة إلى المواد الفعالة سطحياً تعاني من الارتباط مع المذيلات في منطقة السطح 
مسببة التقليل من المساحة السطحية للمجاميع الراسية الأيونية للمواد الفعالة سطحياً، ولا يعتمد ذلك على نوع الكحول [20].
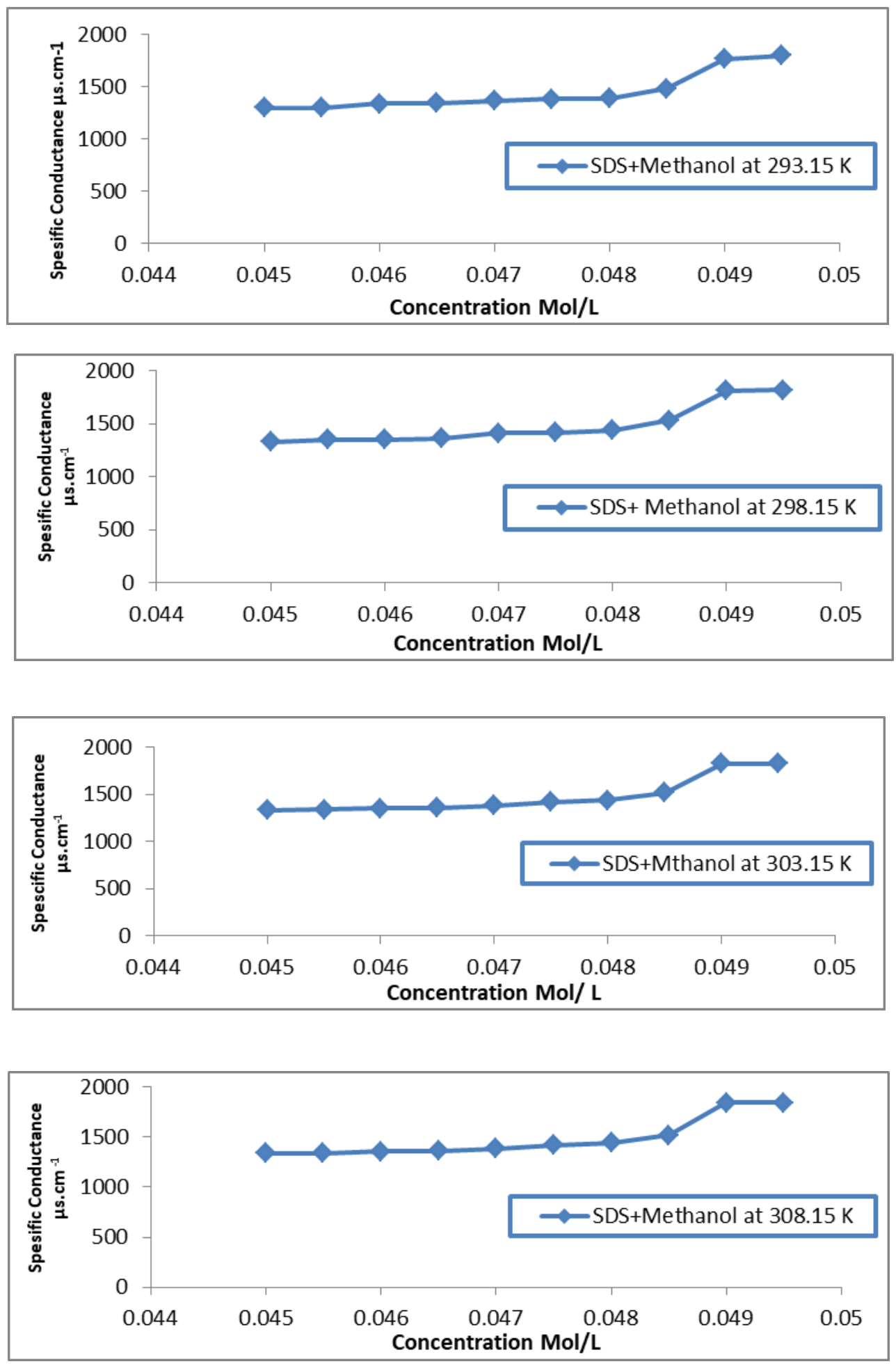

الثكل (5): قياس التوصيلية النوعية مقابل التركيز للمادة الفعالة سطحياً SDS المضاف إليها كحول الميثانول عند درجات حرارية مختلفة 

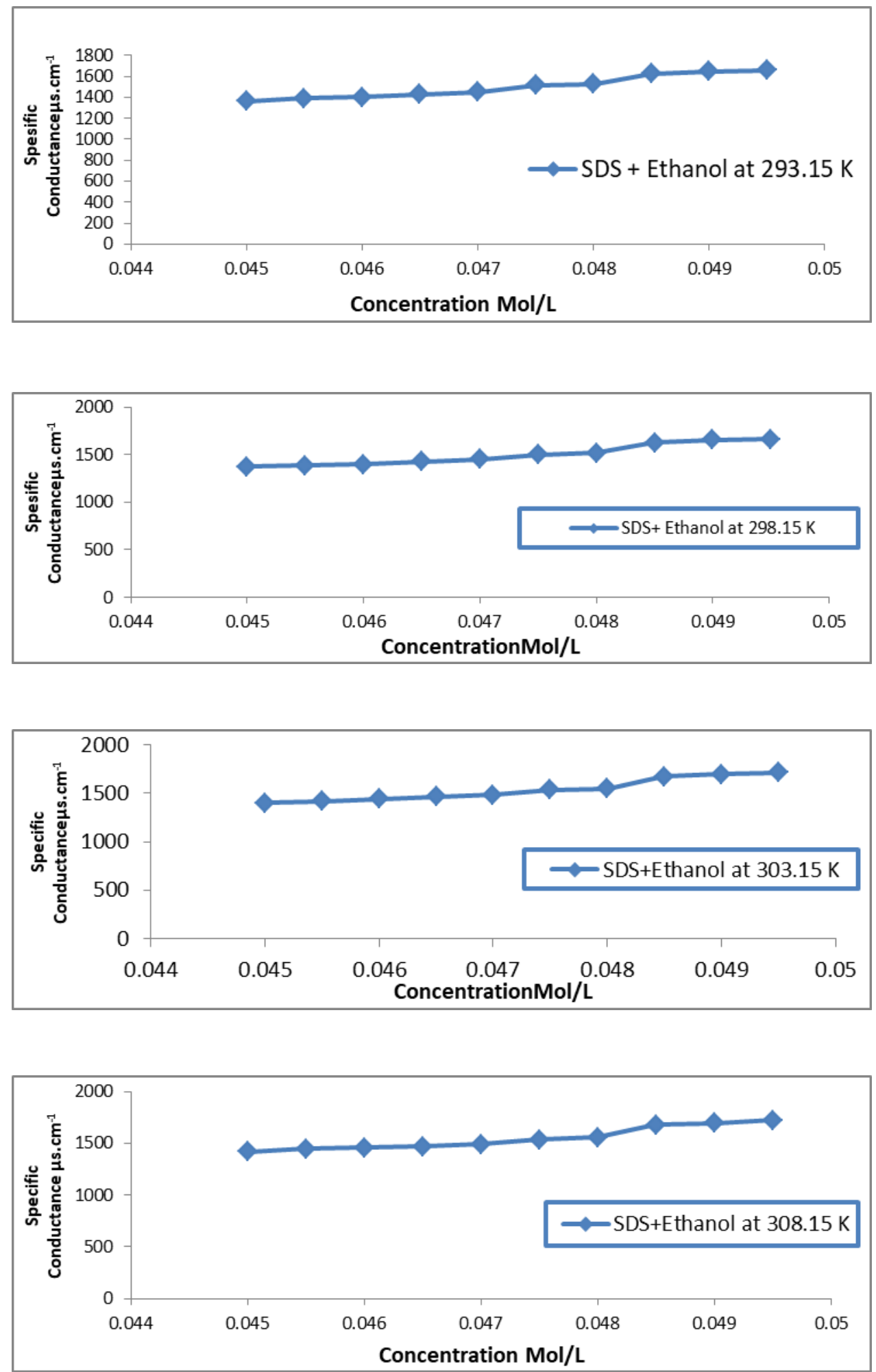

الثكل (6): قياس التوصيلية الكهربائية مقابل التركيز للمادة الفعالة سطحياً SDS المضاف إليها كحول الايثانول عند درجات حرارية مختلفة 

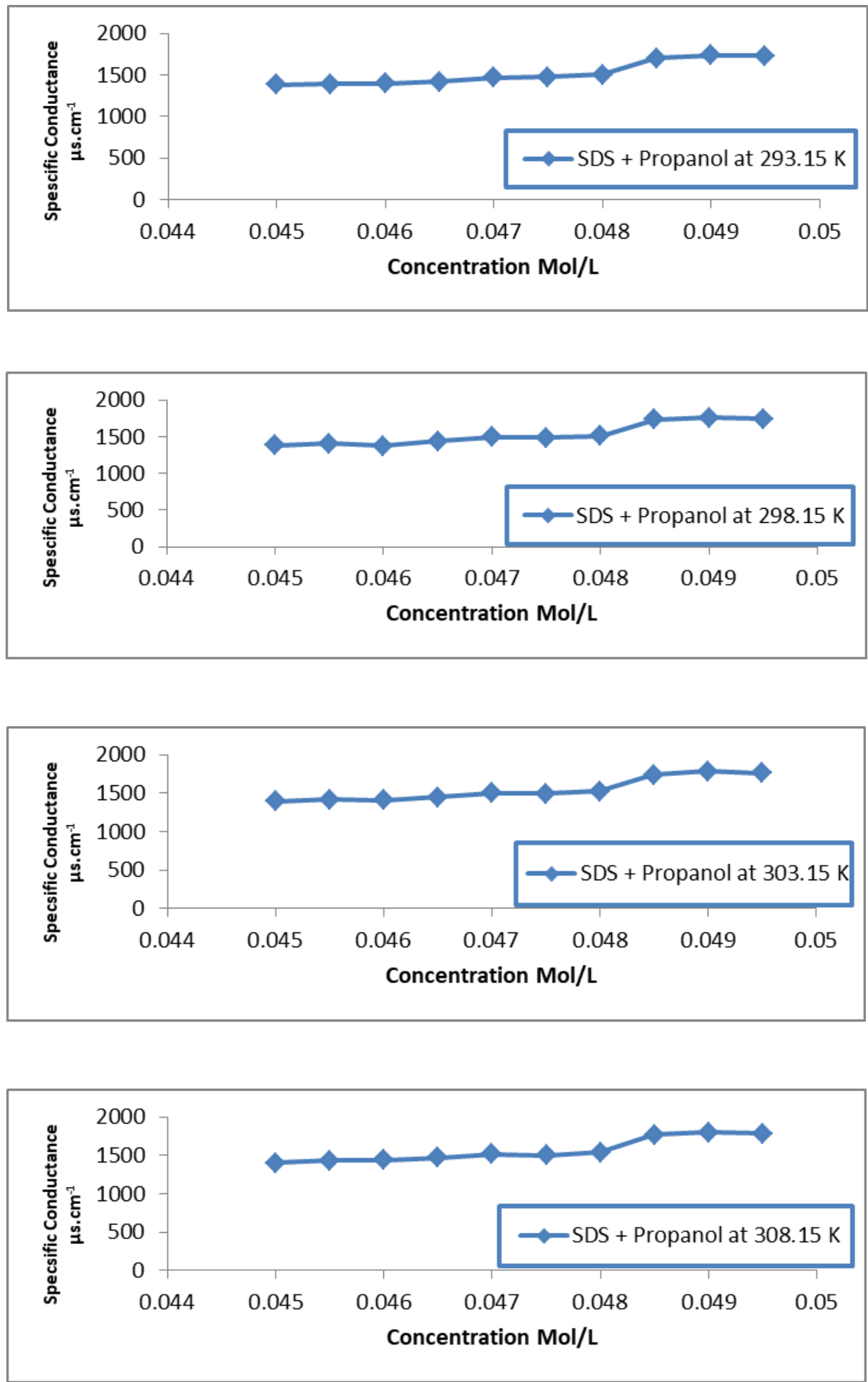

الثكل (7): قياس التوصيلية النوعية مقابل التركيز للمادة الفعالة سطياً SDS المضاف إليها كحول البروبانول عند درجات حرارية مختلفة

يتبين من رسم العلاقة في الأشكال السابقة بين كمية الكحول المضاف (الميثانول) ضد التوصيلية النوعية عند درجة حرارة 293.15k أن قيمة CMC بحدود (0.0480M) والتي تقل تدريجياً بإضافة الايثانول (0.0470M) SDS وهي القيم التي يبدأ عندها تجمع (0.04755M) الدذيلات، إن السبب في ذلك هو أن إضافة الكحولات يؤدي الى زيادة لزوجة المحلول، وبالتالي تكون المذيلات 
الديدانية (Worme Like) [21] وزيادة التشابك بين السلاسل في المحلول، إذ تتغلغل جزيئات الكحول بين جزيئات المذيلات الديدانية مما يؤدي إلى تداخلات بين السلاسل الهيدروكاربونية (تداخل المجاميع الكارهة

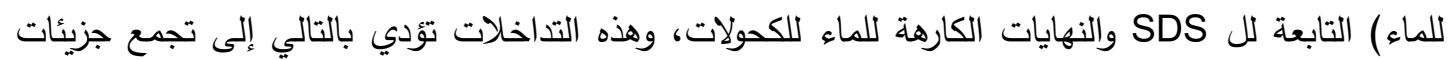
الدذيلات، الذي يؤدي إلى سرعة تكون CMC وانخفاض قيمتها، كما أن للرؤوس القطبية أيضا دوراً في تجمع التهات الجزيئات من خلال التداخل الحاصل بين المجاميع القطبية في SDS ومجموعة الهيدروكسيل للكحولات، وهذها التداخلات تؤدي إلى زيادة التجمع وسرعة تكون المجاميع المحبة للماء وسرعة ظهور

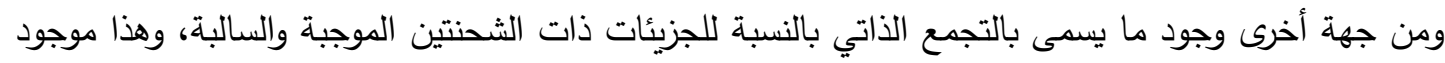

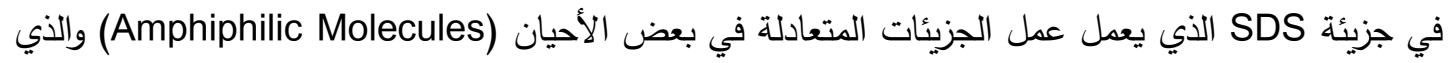
قد يكون موجوداً في حالة SDS حسب رأي بعض الباحثين (6,5) وهذا يقودنا الى ظاهرة أكثر عمقاً هي التداخلات الأيونية المختلفة الموجودة في المحلول، التي لها دور في التجمع المذيلات (Micellization).

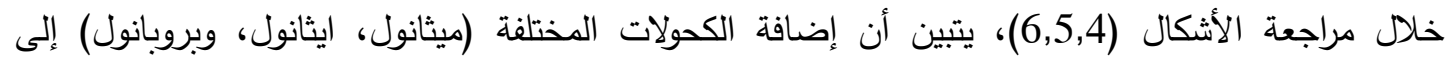
المحلول المائي الممغنط SDS يؤدي الى إنخفاض قيمة CMC. كما هو موضح في الجدول (4) أدناه وذلك الكالك بسبب التداخل المجاميع الكارهة للماء بين السلاسل الهيدروكاربونية للكحولات.

\begin{tabular}{|c|c|c|c|c|c|c|}
\hline $\bar{T} \mathbf{T}(\mathbf{K})$ & $\begin{array}{c}\text { SDS+Methanol } \\
\text { CMC M }\end{array}$ & LnCMC & $\begin{array}{c}\text { SDS+Ethanol } \\
\text { CMC M }\end{array}$ & LnCMC & $\begin{array}{c}\text { SDS+Propanol } \\
\text { CMC M }\end{array}$ & -nCMC \\
\hline $293.15 \mathrm{~K}$ & 0.0480 & -3.0365 & 0.0475 & -3.0470 & 0.0470 & \begin{tabular}{|l|}
-3.0576 \\
\end{tabular} \\
\hline $298.15 \mathrm{~K}$ & 0.0475 & -3.0470 & 0.0465 & -3.0683 & 0.0460 & \begin{tabular}{|l|}
-3.0791 \\
\end{tabular} \\
\hline $303.15 \mathrm{~K}$ & 0.0470 & -3.0576 & 0.0455 & -3.0900 & 0.0450 & -3.1010 \\
\hline $308.15 \mathrm{~K}$ & 0.0465 & -3.0683 & 0.0450 & -3.1010 & 0.0450 & -3.1010 \\
\hline
\end{tabular}

الجدول (4): يوضح قيم CMC للمادة لفعالة سطحياً SDS المضاف لها الكحولات (الميثانول، الايثانول، والبروبانول) عند درجات حرارية مختلفة

إن إضافة كمية قليلة من الكحول العضوي يعطي تغيرات واضحة وملموسة على قيمة CMC للمادة الفعالة

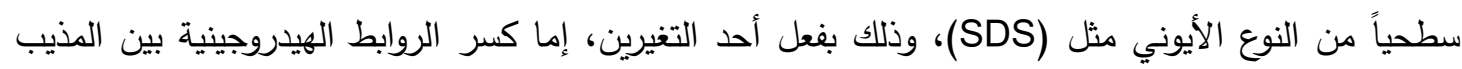

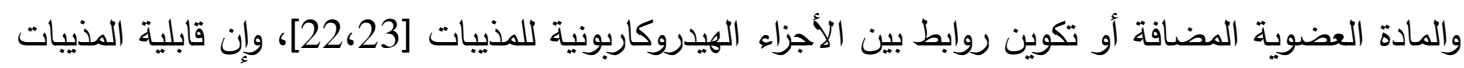
على تكوين روابط هيدروجينية تعتبر مهمة لتكوين المذيلات. لقد تم حساب المتغيرات الثرموداينكية في مدى

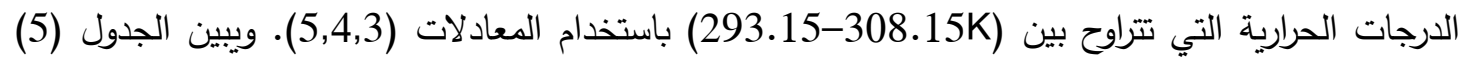

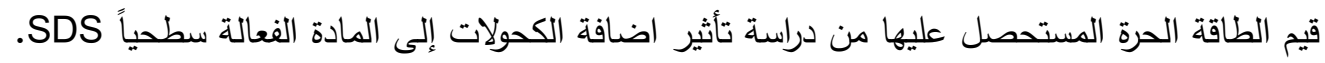

\begin{tabular}{||c|c|c|c||}
\hline \multicolumn{4}{|c||}{$\Delta \mathbf{G}_{\mathbf{m}}^{\mathbf{o}}\left(\mathbf{K J}\right.$. $\left.\mathbf{M o l}^{-\mathbf{1}}\right)$} \\
\hline $\mathbf{T}(\mathbf{K})$ & SDS+Methanol & SDS+Ethanol & SDS+Propanol \\
\hline $293.15 \mathrm{~K}$ & -7.3969 & -7.4224 & -7.4483 \\
\hline $298.15 \mathrm{~K}$ & -7.5491 & -7.6019 & -7.6286 \\
\hline $303.15 \mathrm{~K}$ & -7.7025 & -7.7841 & -7.8118 \\
\hline $308.15 \mathrm{~K}$ & -7.8570 & -7.9407 & -7.9407 \\
\hline \hline
\end{tabular}

الجدول (5): قيم الطاقة الحرة عذد درجات حرارية مختلفة للمادة الفعالة سطحياً SDS المضاف لها الكحولات (الميثانول، الايثانول، والبروبانول) 
وبرسم العلاقة بين LnCMC ودرجات الحرارة اذ يعطي الميل الذي نحسب منه قيمة الانثالبي ونلاحظ انها اعطت علاقة خطية كما في الشكل (8).
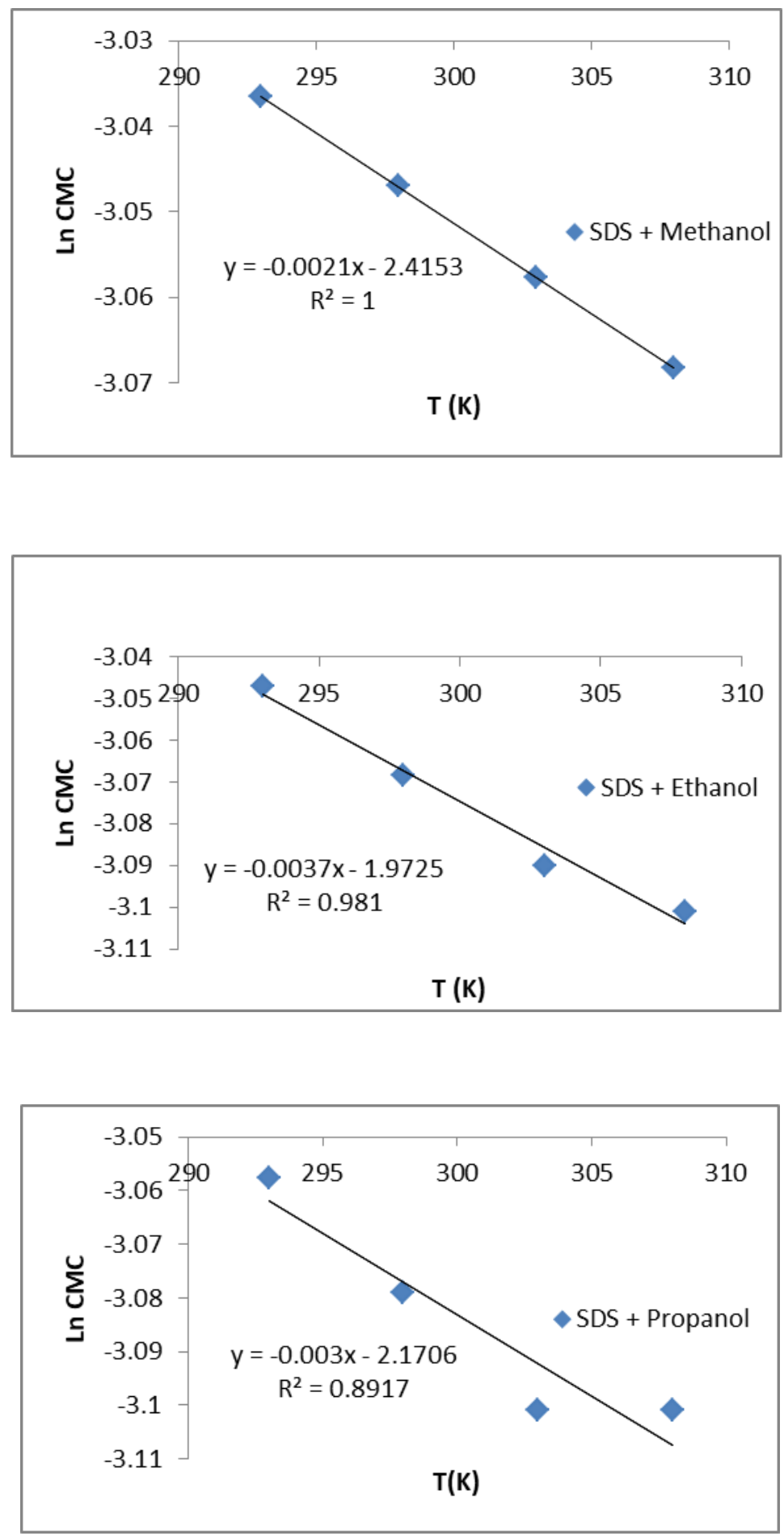

الثكل (8): يوضح LnCMC مقابل درجات الحرارة المختلفة للمادة الفعالة سطحياً SDS المضاف لها الكحولات

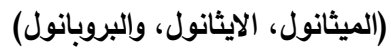


تقدير تركيز ألمذيل الحرج (CMC) لمركب سلفات الدوديسيل في الوسط المائي المفغط باستخدام تقنية التوصيل ...

\begin{tabular}{||c|c|c|c||}
\hline \hline T(K) & SDS+Methanol & SDS+Ethanol & SDS+Propanol \\
\hline $293.15 \mathrm{~K}$ & 1.4988 & 2.6408 & 2.1412 \\
\hline $298.15 \mathrm{~K}$ & 1.5504 & 2.7317 & 2.2149 \\
\hline $303.15 \mathrm{~K}$ & 1.6029 & 2.8242 & 2.2899 \\
\hline $308.15 \mathrm{~K}$ & 1.6562 & 2.9181 & 2.3660 \\
\hline
\end{tabular}

الجدول (6): قيم الانثالبي عند درجات حرارة مختلفة للمادة الفعالة سطحياً SDS المضاف لها الكحولات

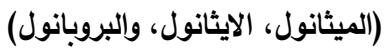

اما قيم الانتروبي الموضحة في الجدول فهي (7):

\begin{tabular}{||c|c|c|c||}
\hline \hline \multicolumn{4}{|c||}{$\Delta \mathbf{S}_{\left(K_{J} . M{ }^{-1} \cdot K^{-1}\right)}$} \\
\hline T (K) & SDS+Methanol & SDS+Ethanol & SDS+Propanol \\
\hline $293.15 ~ K$ & 0.0303 & $\mathbf{0 . 0 3 4 3}$ & $\mathbf{0 . 0 3 2 7 2}$ \\
\hline $298.15 \mathrm{~K}$ & $\mathbf{0 . 0 3 0 5}$ & $\mathbf{0 . 0 3 4 6}$ & $\mathbf{0 . 0 3 3 0 3}$ \\
\hline $303.15 \mathrm{~K}$ & $\mathbf{0 . 0 3 0 7}$ & $\mathbf{0 . 0 3 5 0}$ & $\mathbf{0 . 0 3 3 3 3}$ \\
\hline $308.15 \mathrm{~K}$ & $\mathbf{0 . 0 3 0 8}$ & $\mathbf{0 . 0 3 5 2}$ & $\mathbf{0 . 0 3 3 4 6}$ \\
\hline \hline
\end{tabular}

الجدول (7): قيم الاتتروبي عند درجات حرارية مختلفة للمادة الفعالة سطحياً SDS المضاف لها الكحولات

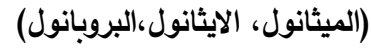

إذ رسمت المتغيرات الثرموداينمكية عند درجات حرارية مختلفة للمادة الفعالة سطحياً المضاف لها كحول الميثانول كما موضح هو في الثكل (9):
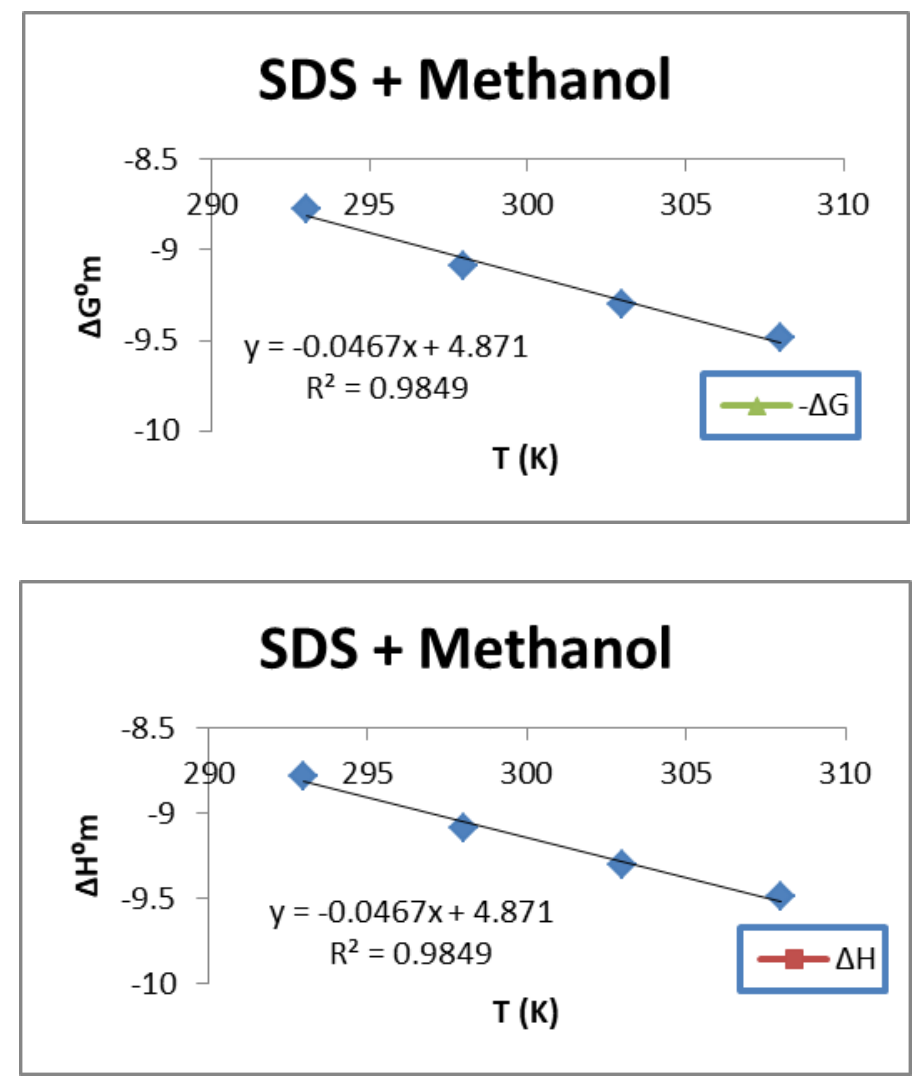


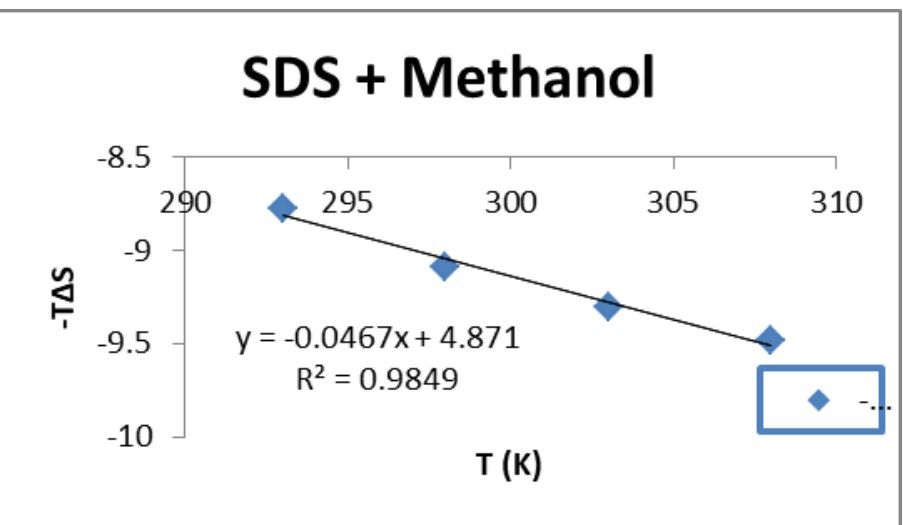

الثكل (9): المتغيرات الثرموداينمكية عند درجات حرارية مختلفة للمادة الفعالة سطحياً المضاف لها كحول الميثانول

إذ رسمت المتغيرات الثرموداينمكية عند درجات حرارية مختلفة للمادة الفعالة سطحياً المضاف لها كحول الايثانول كما موضتح هو في الشكل (10):
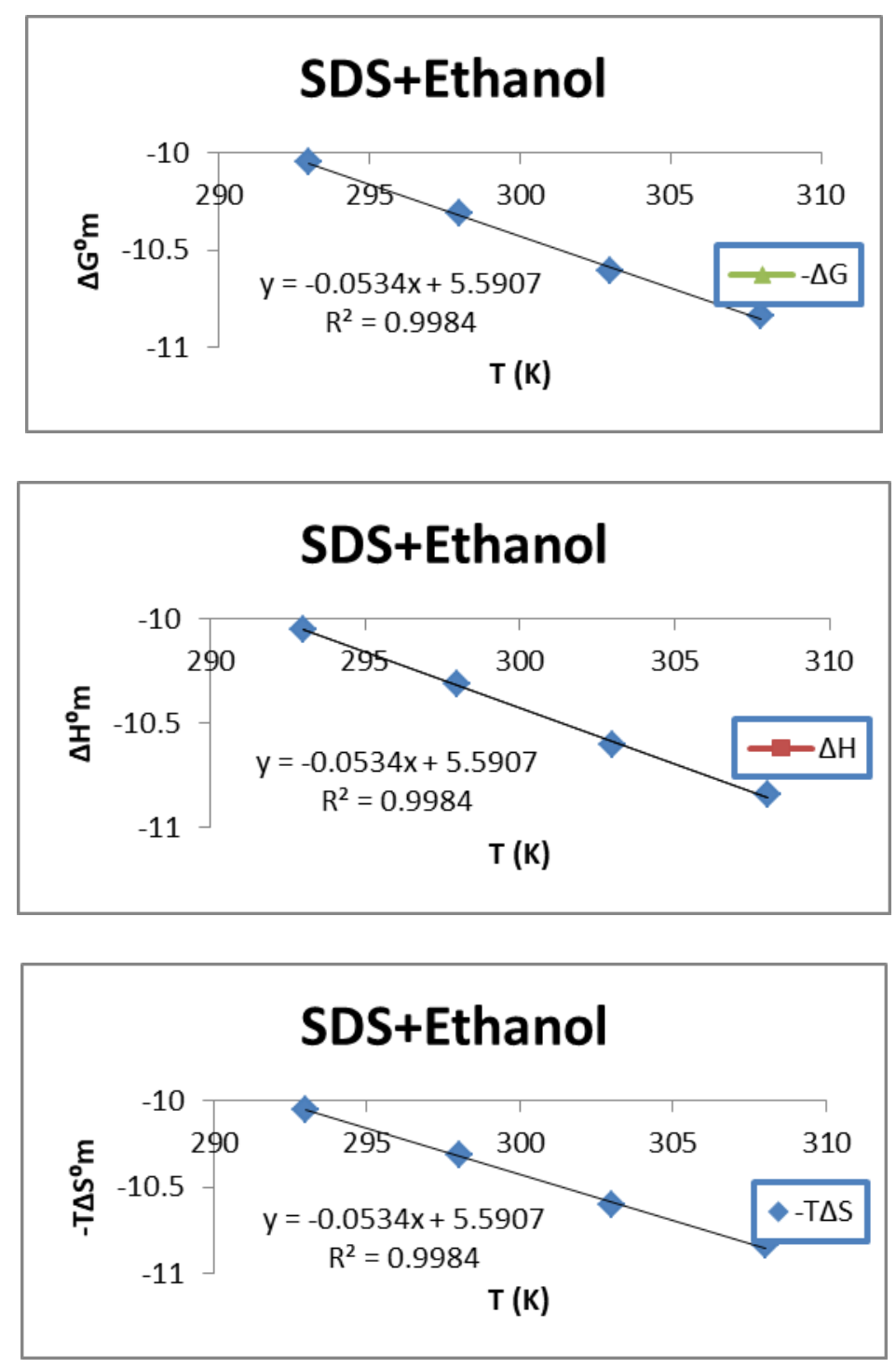

الثكل (10): المتغيرات الثرموداينمكية عند درجات حرارية مختلفة للمادة الفعالة سطحياً المضاف لها كحول الايثانول 
إذ رسمت المتغيرات الثرموداينمكية عند درجات حرارية مختلفة للمادة الفعالة سطحياً المضاف لها كحول البروبانول كما موضح هو في الشكل (11):
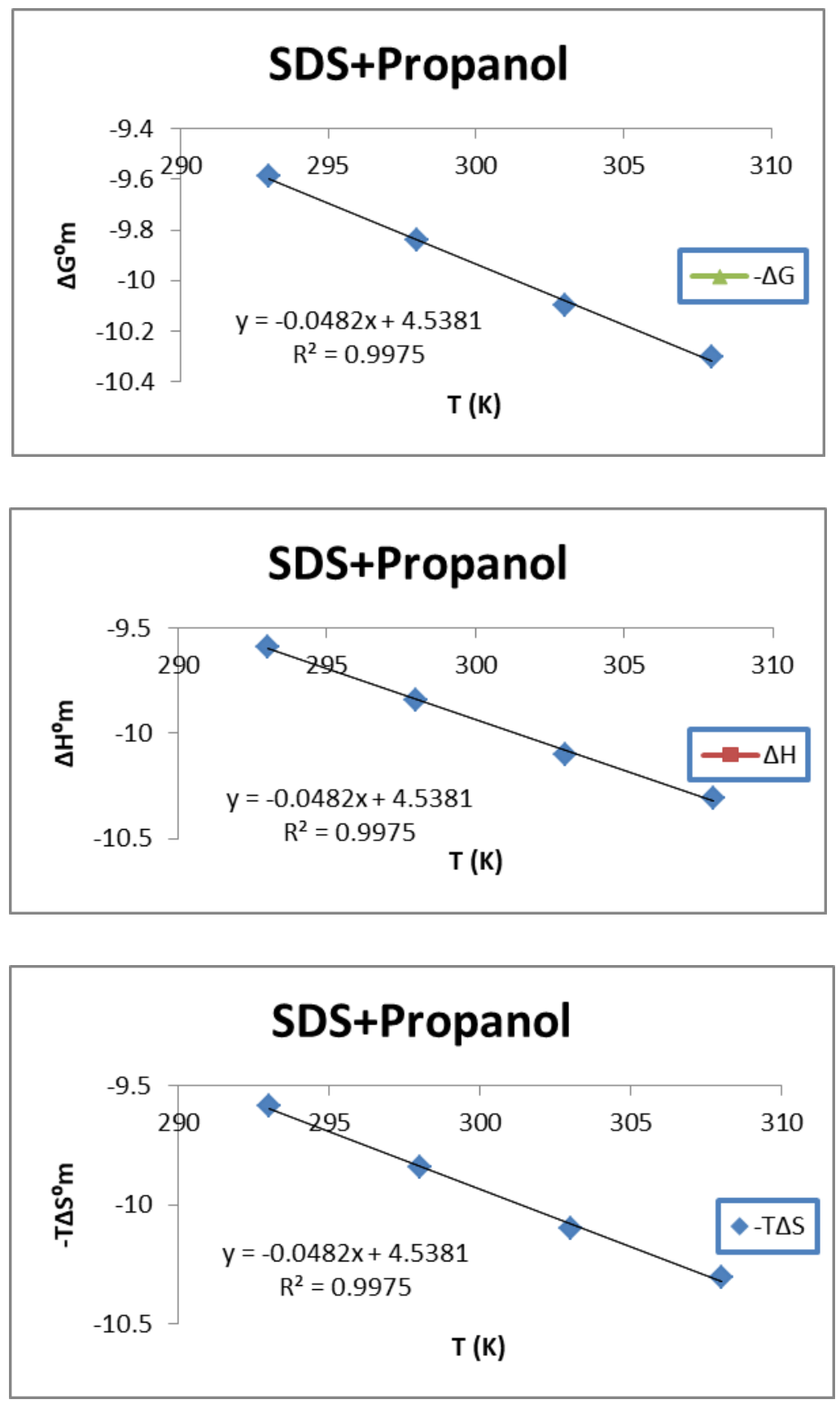

الثكل (11): المتغيرات الثرموداينمكية عند درجات حرارية مختلفة للمادة الفعالة سطحياً SDS المضاف لها كحول البروبانول

المناقشة:

إن تجمع المذيلات (Micellization) يتأثر دائماً بعدة عوامل منها: طبيعة المادة الفعالة سطحياً من حيث طول السلسلة الهيدرو كربونية والمجموعة الرأسية، ودرجة الحرارة، والضغط، والقوة الأيونية، والدالة

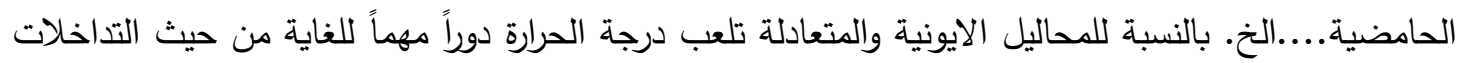


الكارهة للماء والمجموعة الرأسية المحبة للماء القطبية. وبالنسبة للمحاليل غير الايونية فإن CMC تتخض بزيادة درجة الحرارة نظراً لازدياد التأثير الكاره للماء بسبب تحطم الروابط الهيدروجينية بين المجاميع الرأسية وجزيئات الماء في حالة المركبات الأيونية، فإن CMC تتخفض إلى حد معين ثم ترتفع قيمتها عند الدرجات الحرارية

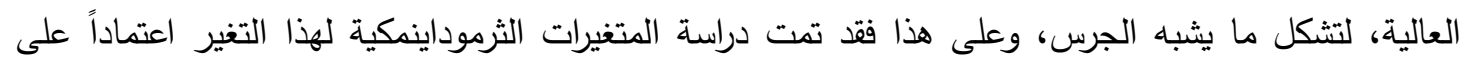

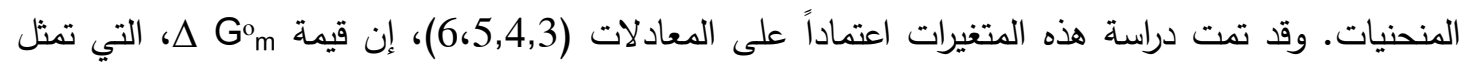

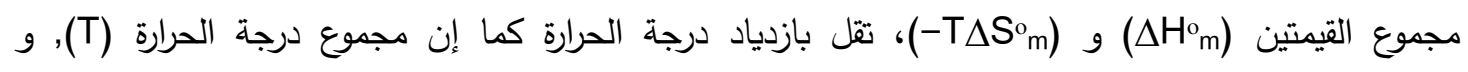

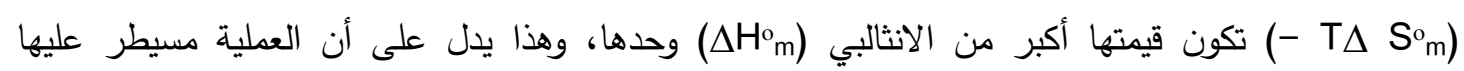

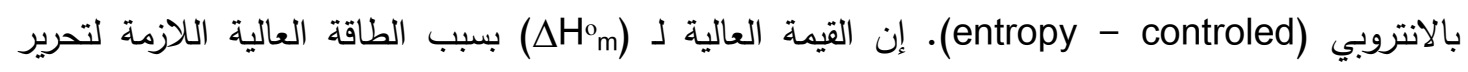
جزيئات المذيب من الطبقة المائية المحيطة بالمذيلات المسؤولة عن المجاميع المحبة للماء المتواجدة في الجزيئة المتعادلة (Amphiphilic molecule) التابعة للمادة الفعالة سطحيا (Surfactant). إضافة الى ذلى ذلك هنالك طاقة تتحرر نتيجة لارتباط المذيلات بالسلاسل الهيدروكاربونية الموجودة في المحلول وإعادة تكوين الروابط

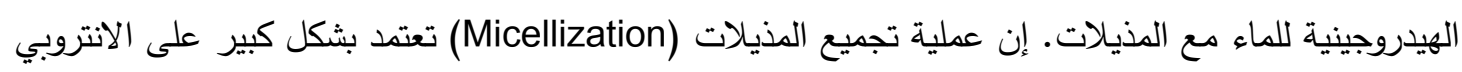

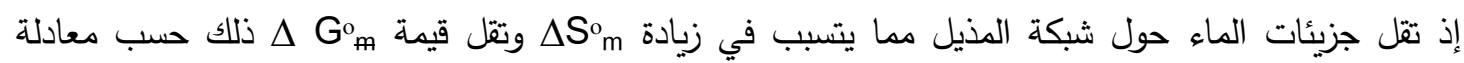

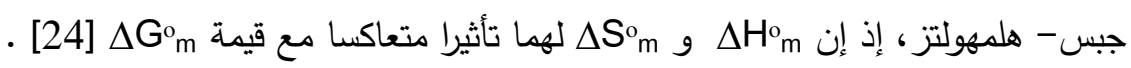

\section{References}

1) Rossoff, S. (1974). Handbook and Veternary Drugs. New york: Springer Publishing Comypany, P 542.

2) Mosquera, M.G.; Varewla, L.M; Nalwa, H.S. (2001). Association colloids:cmc apropertyto calculate, hand book of surface and interfaces of materials Acacemic press New York, P 401.

3) Shah, S.S.; Laghari, G.M.; Naeem, K.; and Shah, S.W.H. (1998). Colloids Surf., A, PP 143, 111.

4) Naseem, B.; Sabri, A.; Hasan, A.; and Shah, S.S. Colloids Surf., B, 2004; 35:7.

5) Gunnrsson, G.; Jonsson, B.; and Wennerstrom, H. J Phys Chem., 1980; 84:3114 $-3121$.

6) Kundryashov, E.; Kapustina, T.; Morrissey, S.; Bucking, V.; and Dawson, K. J. (1998). Colloid Interface Sci., 203(1):59-68.

7) PATIL, T.J. and Mudawadkar, A.D. (2016). Conductometric Studies of Micellization of Sodium Dodecyl Sulfate in Presence of Non- Polar Additives at Various Temperatures, 5(1):14.

8) Dixit, S.B.; Bhasin, R.; Rajasekaran, E.; and Jayaram, B. J. Chem Soc Faraday Trans., 1997; 93:1105-1113.

9) Lehinger, A.L, Nelson, D.L, and Cox M.M. Worth Publishers, USA, 1993.

10) Mayers, D. Surfactant in Cosmetic, Marcel Dekker Ing., New york, 1997; 47.

11) Langeven,V. (1994). Micelle and micro emulsions Annual Review of physical chemistry; 43:341-369.

12) Moulik, S., Paul, B. (2001). Uses and Application of micro emulsions Current Science, 80:990-1001.

13) Chanrasiya, R. and Habbar, U. (2017). Micelle of Nanoparticle Synthesis and Biomoleccule Separation-Nanoscience in food and Agrcal, 4(24):181-211. 
14) Al-Dabbagh, Abdul Majid and Aqraoui, Banan. (1992). Al-Harikiat and Electro- Chemistry, Dar al-Kitab for Printing and Publishing, Mosul University, Mosul, Iraq, 640-625

15) Dalmas, P. "Conductivity Theory and Practice", Radiometer Anlytical SAS France. 2004.

16) Ramachandran, H. and K,S.D.K. (2018). An Experimental Study on the Use of Magnetized Water in Concrete with M Sand as Fine Aggregate, Journal of Engineering, 8(6): 26-32

17) Nouri, Solaf Adnan. (2016). Aspects of they use of Magnetized Water technology in agriculture and public health in Iraq. Journal of the Faculty of basic Education University of Mustansiriya, 22(94).

18) Chetouani, A.; Tennouga, L.; Mansri1, A.; Medjahed, K.; and Warad, I. (2015). The micelle formation of cationic and anionic surfactants in aqueous medium: Determination of CMC and thermodynamic parameters at different temperatures; 6(10):2711-2716.

19) Chodzińska, A.; Zdziennicka, A.; and Jańczuk, B. (2012) Volumetric and Surface Properties of Short Chain Alcohols in Aqueous Solution-Air Systems at 293 K, J Solution Chem.; 41(12): 2226-2245.

20) Acar, G.K. and Sidim, T. (2013). Alcohols Effect on Critic Micelle Concentration of Polysorbate 20 and Cetyl Trimethyl Ammonium Bromine Mixed Solutions,16(4):601-607 .

21) Khalil, R. A. and Alsamarrai, L.H. (2015). Study The Effect of Ethanol on The Formation of The Wormlike Micelle For a Mixture of Ionic Surfactants; 12:2117-2121.

22) Chetouani, A. ;Tennouga, L.; Mansri1, A.; Medjahed, K.; and Warad, I.(2015). The micelle formation of cationic and anionic surfactants in aqueous medium: Determination of $\mathrm{CMC}$ and thermodynamic parameters at different temperatures, 6(10): 2711-2716.

23) Demissie, H. and Duraisamy H.(2016). Effect of Electrolyte on The Surface and Micellar Characteritics of Sodium Dodecyl Sulphate Surfactant Solution, J.Sci.Inno.Res, 5(6):208-214.

24) Nowicki, J.; Tuczak, J.; and Stanczyk, D.(2016). Dual Functionality of Amphiphilic 1-alkyl-3-methylimidazolium hydrogen sulfate ionic liquids surfactant with catalytic function; 6:11591-11601. 